\title{
Bicruciate-retaining Total Knee Replacement Provides Satisfactory Function and Implant Survivorship at 23 Years
}

\author{
James W. Pritchett MD
}

Received: 24 September 2014/ Accepted: 18 February 2015/Published online: 27 February 2015

(C) The Association of Bone and Joint Surgeons (B) 2015

\begin{abstract}
Background One of the goals of a TKA is to approximate the function of a normal knee. Preserving the natural ligaments might provide a method of restoring close to normal function. Sacrifice of the ACL is common and practical during a TKA. However, this ligament is functional in more than $60 \%$ of patients undergoing a TKA and kinematic studies support the concept of bicruciateretaining (that is, ACL-preserving) TKA; however, relatively few studies have evaluated patients treated with bicruciate-retaining TKA implants.

Questions/purposes I asked: (1) what is the long-term (minimum 20-year) survivorship, (2) what are the functional results, and (3) what are the reasons for revision of bicruciate-retaining knee arthroplasty prostheses?

Methods From January 1989 to September 1992, I performed 639 total knee replacements in 537 patients. Of

Each author certifies that he or she, or a member of his or her immediate family, has no funding or commercial associations (eg, consultancies, stock ownership, equity interest, patent/licensing arrangements, etc) that might pose a conflict of interest in connection with the submitted article.

All ICMJE Conflict of Interest Forms for authors and Clinical Orthopaedics and Related Research ${ }^{\mathbb{R}}$ editors and board members are on file with the publication and can be viewed on request. Clinical Orthopaedics and Related Research ${ }^{\mathbb{R}}$ neither advocates nor endorses the use of any treatment, drug, or device. Readers are encouraged to always seek additional information, including FDAapproval status, of any drug or device prior to clinical use.

The author certifies that his institution approved the human protocol for this investigation, that all investigations were conducted in conformity with ethical principles of research, and that informed consent for participation in the study was obtained.
\end{abstract}

J. W. Pritchett $(\square)$

901 Boren Avenue, \#900, Seattle, WA 98104, USA

e-mail: bonerecon@aol.com these, 489 were performed in 390 patients using a bicruciate-retaining, minimally constrained device. During the period in question, this knee prosthesis was used for all patients observed intraoperatively to have an intact, functional ACL with between $15^{\circ}$ varus and $15^{\circ}$ valgus joint deformity. There were 234 women and 156 men with a mean age at surgery of 65 years (range, 42-84 years) and a primary diagnosis of osteoarthritis in $89 \%$. The patella was resurfaced in all knees. The mean followup was 23 years (range, 20-24 years). At the time of this review, 199 (51\%) patients had died and $31(8 \%)$ patients were lost to followup, leaving 160 (41\%) patients (214 knees) available for review. Component survivorship was determined by competing-risks analysis and Kaplan Meier survivorship analysis with revision for any reason as the primary endpoint. Patients were evaluated every 2 years to assess ROM, joint laxity, knee stability, and to determine American Knee Society scores.

Results The Kaplan-Meier survivorship was 89\% (95\% CI, 82\%-93\%) at 23 years with revision for any reason as the endpoint. Competing-risks survivorship was $94 \%(95 \%$ CI, 91\%\%-96\%) at 23 years. At followup, the mean age of the patients was 84 years (range, 63-101 years), the mean flexion was $117^{\circ}$ (range, $90^{\circ}-130^{\circ}$ ), the mean American Knee Society score improved from a preoperative mean of 42 (range, 26-49) to 91 (range, 61-100; $\mathrm{p}<.001$ ). Twenty-two knees in 21 patients $(5.6 \%)$ were revised, most commonly because of polyethylene wear.

Conclusions ACL sacrifice may be an unnecessary concession during TKA. This study found satisfactory survivorship and function after more than 20 years of use for patients receiving a bicruciate-retaining TKA implant. A TKA that preserves cruciate ligaments provides a stable, well-functioning knee with a low likelihood of revision at long-term followup. Retaining both cruciate ligaments 
during knee arthroplasty is an attractive concept that is worth considering.

Level of Evidence Level IV, therapeutic study.

\section{Introduction}

Normal knee function relies on smooth, uninterrupted motion, which in turn depends on stable, well-lubricated, low-friction articular surfaces. Knee replacement involves compromises between stability and flexibility, and historically has included removal of one or both cruciate ligaments. As an alternative to this philosophy, a bicruciate-retaining knee prosthesis was developed in 1971 [24]. The design emphasized preserving the ligaments, minimizing bone resection, and limiting constraint with the goal of allowing more natural movement of the knee compared with other prostheses. AP stability was provided by the preserved cruciate ligaments rather than the shape of the polyethylene.

Although the ACL is functional in more than $60 \%$ of patients undergoing a TKA $[1,7,8]$ and kinematic studies support the concept of bicruciate-retaining (that is, ACLpreserving) TKA [14, 23], few studies have evaluated patients treated with bicruciate-retaining TKA implants [16-19, 21]. Because of these perceived advantages, I used a bicruciate-retaining TKA implant in my practice for all patients with an intact ACL and acceptable deformity during a 3-year period, and because of the limited number of studies available at longer term [21], I sought to evaluate their performance and durability.

I asked: (1) what is the long-term (minimum 20-year) survivorship, (2) what are the functional results, and (3) what are the reasons for revision of a bicruciate-retaining knee arthroplasty?

\section{Patients and Methods}

From January 1989 to September 1992, I performed 639 total knee replacements in 537 patients. Of these, 489 (77\%) were performed in 390 patients using a bicruciateretaining, minimally constrained device (Townley Anatomic, Biopro Inc, Port Huron, MI, USA) (Fig. 1). Patients selected for a TKA with a bicruciate-retaining prosthesis had no history of ACL insufficiency. During the period in question, this prosthesis was used for all patients observed intraoperatively to have an intact, functional ACL and limited deformity. There were 156 men (40\%) and 234 women $(60 \%)$ with a mean age at surgery of 65 years (SD, 8.6; range, 42-84 years). The primary diagnosis was osteoarthritis in $347(89 \%)$ knees and inflammatory arthritis or osteonecrosis in $43(11 \%)$. At the time of the surgery, the ACL was functionally intact as shown by testing with a probe and by negative Lachman, pivot shift, and anterior drawer tests. The appearance and integrity of the ACL also was tested before and after placing the implants. For patients without an ACL and in patients with greater than $15^{\circ}$ valgus or $15^{\circ}$ varus, I used a posterior cruciate or posterior stabilized prosthesis. The final decision regarding implanting a bicruciate-retaining prosthesis was made intraoperatively.

The surgical technique followed the basic principles of ligamentous balancing. Neither the ACL nor the PCL was recessed. The tibia was prepared with $6^{\circ}$ of posterior slope. Initially, alignment and balance in extension were achieved by correcting the coronal deformity with appropriate capsular and ligamentous releases. The femur was prepared first and the tibia was not subluxed forward on the femur during surgery. The tibial osteotomy was performed in flexion in $2^{\circ}$ to $3^{\circ}$ varus with respect to the mechanical axis of the knee $[12,24]$. The tibial spines and insertions of the cruciate ligaments were left in continuity with the rest of the tibia. The tibial component was placed in slight external rotation following the orientation of the fibers of the ACL [21]. A complete ROM test was performed with trial implants in place. There were two intraoperative complications consisting of two instances of tibial eminence fracture as the knee was moved from flexion to extension during trial ROM testing. This occurred because the knee was too tight owing to insufficient distal femur or proximal tibial resection, or incomplete ligament balancing. The eminence was repaired with a screw, the knee was rebalanced, and the procedure was completed. There were no ligament ruptures during surgery. These procedures were performed by one surgeon (JWP) with many years of experience performing TKAs with implantation of a bicruciate-retaining prosthesis.

The femoral component is cobalt-chromium with an asymmetric trochlear groove requiring right and left components. The tibial component consists of a flat symmetric polyethylene (PE) insert on a single-piece horseshoeshaped titanium tibial tray with two round fixation pegs. The prosthesis is minimally constrained. One-piece tibial PE inserts 8 to $11 \mathrm{~mm}$ thick were used for all patients. Conventional 1050 ram-extruded PE sterilized in ethylene oxide was used. Patellar resurfacing was performed for all patients with a dome-shaped PE patellar component. Implants were placed with cement [24].

Range of knee movement was assessed with a goniometer, laxity was assessed manually, and American Knee Society knee scores were completed at each visit [13]. Patients were followed every 2 years with The Knee Society Score ${ }^{\circledR}$. The knee score at the end of the second year was used to compare with the preoperative score. 


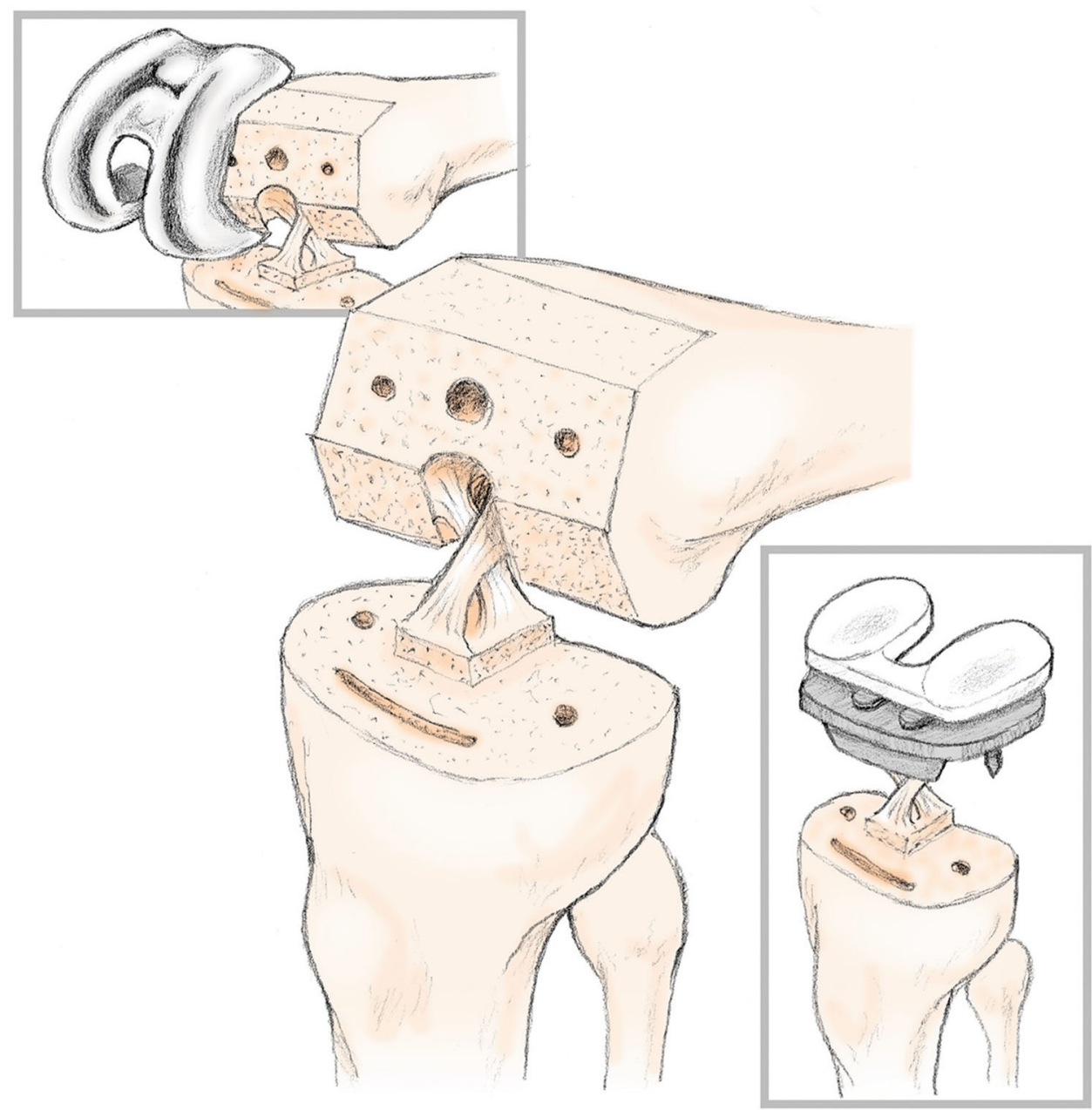

Fig. 1 These drawings show the prepared femur and tibia including the retained tibial eminence and both cruciate ligaments. The femoral and two-piece tibial component is shown with the prepared slot in the tibia for the keel and pilot holes for the spikes of the tibial component.

Standing AP (Fig. 2A) and lateral (Fig. 2B) radiographs of the affected knee were taken at each followup to look for signs of implant failure such as wear and loosening. The orientation of the prosthesis relative to the anatomic axis of the knee was measured on the AP and lateral radiographs.

Patients were followed every 2 years for a minimum of 20 years after the surgery (range, 20-24 years; mean, 23 years). At the time of this review, 199 (51\%) patients had died (237 knees) and 31 (8\%) (37 knees) were lost to followup, leaving 160 (41\%) (214 knees) with a mean age of 84 years (range, 65-101 years) at followup.

The implant survival rate was determined by a competing-events and Kaplan-Meier survivorship analysis with 95\% CIs with revision for any reason as the endpoint $[4,9$, 10]. Comparisons were tested using the Mann-Whitney U test and chi-square analysis where appropriate. A p value less than 0.05 was considered statistically significant. SPSS Version 20 (SPSS Inc, Chicago, IL, USA) was used for the statistical analyses.

\section{Results}

The Kaplan-Meier survivorship analysis at a mean followup of 23 years was $89 \%$ (95\% CI, 82\%-93\%) with revision for any reason as the endpoint (Fig. 3). Competing-risk survivorship at 23 years was $94 \%$ (95\% CI, 91\%96\%) (Fig 4).

Patients showed improvements in preoperative to postoperative flexion and Knee Society scores (Table 1). Flexion improved from a preoperative mean of $104^{\circ}$ to $117^{\circ}$ postoperatively $(\mathrm{p}<0.001)$ and Knee Society scores improved from a preoperative mean of 42 (range, 26-49) to 91 (range, 61-100) postoperatively ( $\mathrm{p}<0.001)$.

Twenty-two knees in 21 patients were revised (Table 2). The mean time from implantation to revision was 12 years (range, 5-22 years). No difference was found between the group of patients with revised implants and the group with surviving implants for primary diagnosis, mean age, mean BMI, mean postoperative tibiofemoral axis, mean posterior slope of the tibial tray, mean ROM, and stability. 
The most common reason for revision was PE wear and this was the sole reason for revision in seven knees. The PE was exchanged in these seven knees. Two knees that were unstable and one that was stiff also were treated by PE exchange. The revision procedure consisted of exchange of only the PE insert in 10 patients. Femoral and tibial component revision was performed in four knees, tibial component revision was performed in three knees, and the patellar component was revised in one knee. The mean PE
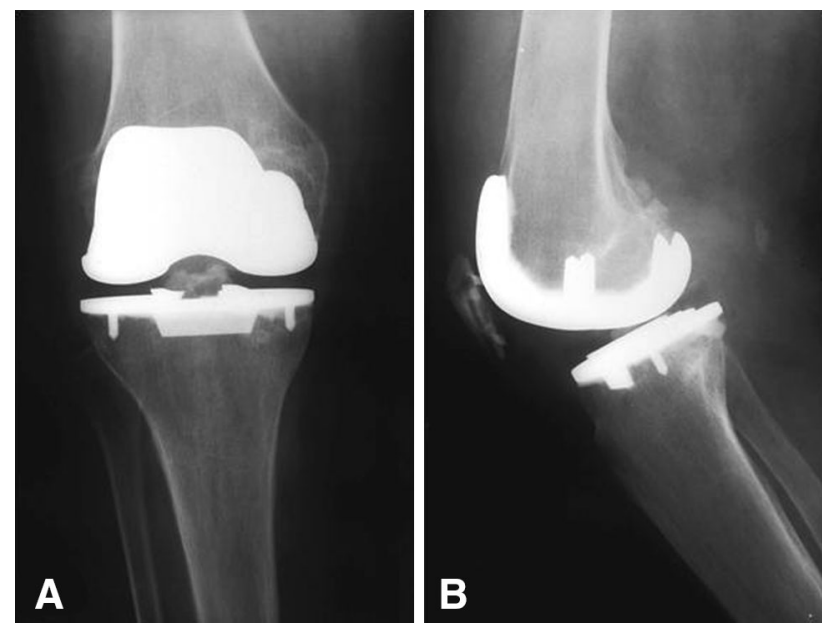

Fig. 2A-B (A) AP and (B) lateral radiographs show the bicruciateretaining total knee prosthesis 20 years after insertion. There is a cemented all polyethylene patellar prosthesis and the femoral and tibial prostheses are cemented. The preserved tibial eminence is seen in the center of the radiograph. The spikes and keel of the tibial component are shown. There is no sign of component loosening or polyethylene wear. thickness in revised knees was $11 \mathrm{~mm}$ (range, 8-14 mm). Osteolysis was seen in seven knees during revision surgery. The components in three knees were revised to stemmed components. In four knees the areas of osteolysis at surgery were 4 to $7 \mathrm{~mm}$ and in three knees the areas were 8 to $12 \mathrm{~mm}$. The ACL and PCL were present during revision surgery in all but one knee.

\section{Discussion}

The ACL is commonly sacrificed during TKA, but there is some evidence that retention of the ACL results in superior kinematics after surgery [14, 22, 23]. During the early development of total knee implants, several prostheses were designed specifically to retain the ACL and PCL [21, 24]. Between 1989 and 1992, I used a bicruciateretaining implant for patients undergoing TKA who had an intact ACL and acceptable deformity. Because of the paucity of long-term data regarding bicruciate-retaining total knee implants, I sought to assess the results of procedures at least 20 years later. I posed the following questions: (1) What is the long-term survivorship of this bicruciate-retaining knee replacement? (2) What are the functional results of this bicruciate-retaining knee replacement? (3) What are the reasons for revision of bicruciate-retaining knee replacement?

This study has limitations. As with other studies of TKAs with followups greater than 20 years, the number of patients who died is high yet similar to the mortality rate of a comparable population without knee problems [6, 15, 20,21]. At followup, the mean age of the patients was 84 years and

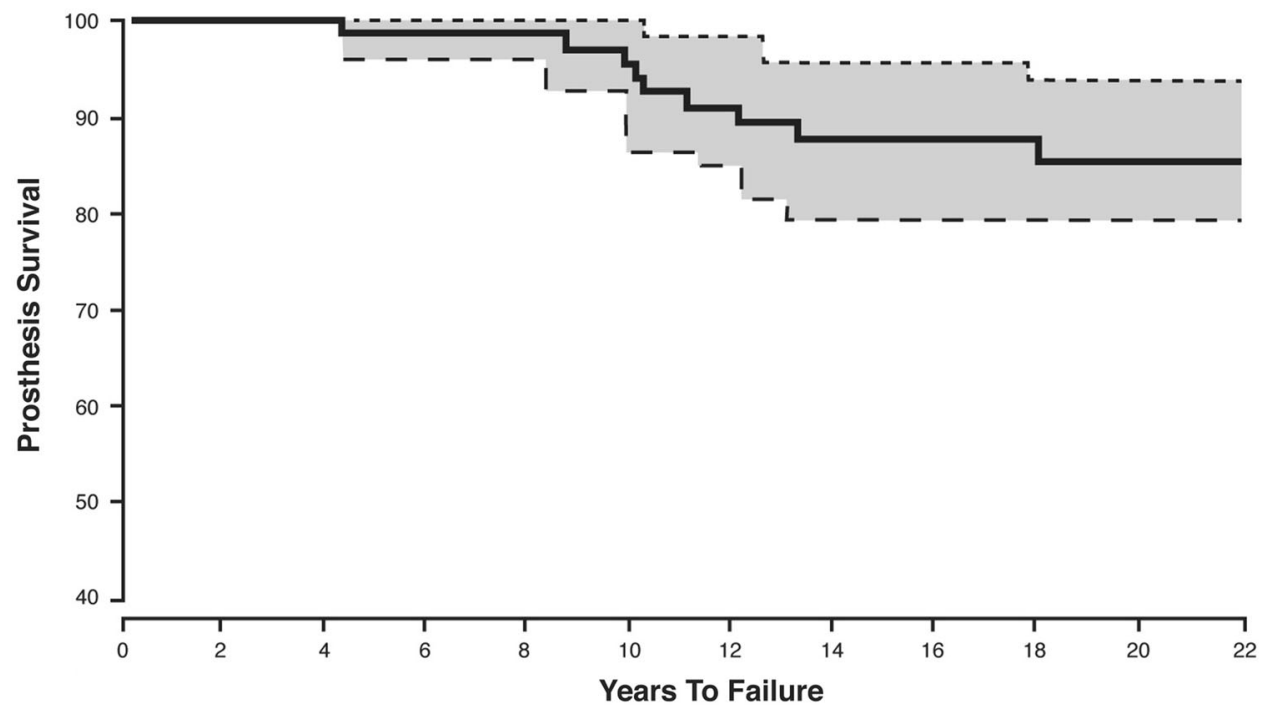

Fig. 3 A Kaplan-Meier survivorship curve with revision for any reason as the endpoint with 95\% CIs (dotted lines) is shown. 


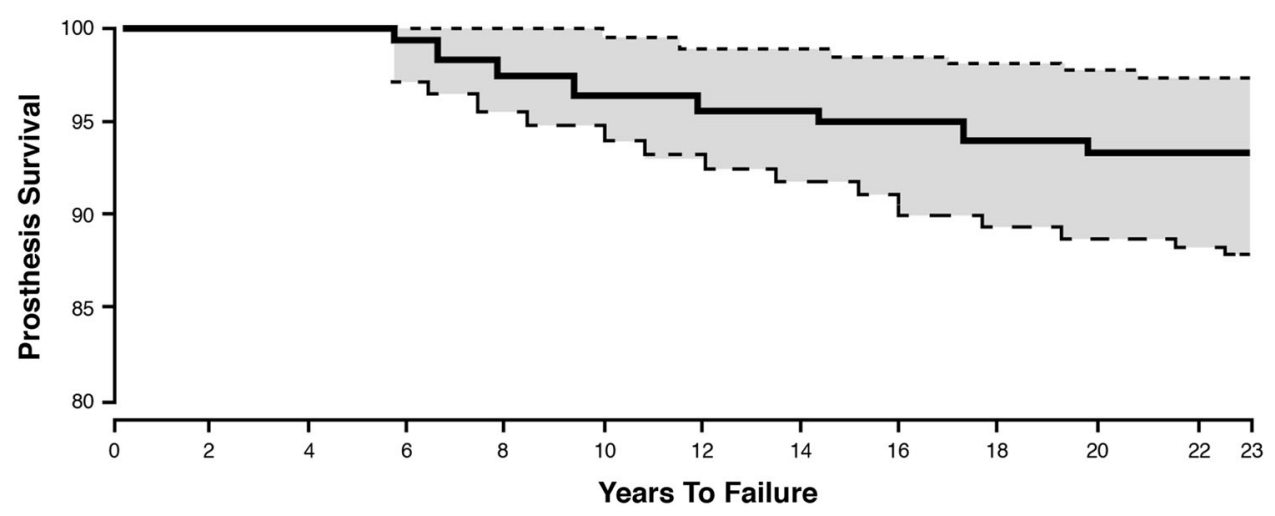

Fig. 4 A competing-risk survivorship curve with revision for any reason as the endpoint with 95\% CIs (dotted lines) is shown.

Table 1. Mean pre- and postoperative functional scores for 160 patients (214 knees)

\begin{tabular}{|c|c|c|c|}
\hline Variable & Preoperative & Postoperative & $\mathrm{p}$ value \\
\hline Flexion $^{\circ}$ (range) & $104(10-130)$ & $117(90-130)$ & $<0.001$ \\
\hline American Knee Society score (range) & $42(26-49)$ & $91(61-100)$ & $<0.001$ \\
\hline Mediolateral laxity $\left({ }^{\circ}\right.$; range $)$ & $1.0(0-5)$ & $0.5(0-4)$ & $>0.01$ \\
\hline Tibiofemoral angle $\left({ }^{\circ}\right.$; range $)$ & $-1(-15$ to 15$)$ & $4(1-8)$ & $<0.001$ \\
\hline Posterior tibial slope $\left({ }^{\circ}\right.$; range) & $5(2-9)$ & $6(3-9)$ & $>0.01$ \\
\hline
\end{tabular}

Table 2. Indication(s) for revision of 22 knees (21 patients)

\begin{tabular}{lll}
\hline Indication(s) & $\begin{array}{l}\text { Knees } \\
(\mathrm{n})\end{array}$ & Additional information \\
\hline $\begin{array}{l}\text { Polyethylene wear } \\
\begin{array}{l}\text { Polyethylene wear }+ \\
\text { tibial component } \\
\text { fracture }\end{array}\end{array}$ & 1 & 1 ACL rupture \\
$\begin{array}{l}\text { Polyethylene wear }+ \\
\text { aseptic loosening }\end{array}$ & 1 & \\
$\begin{array}{l}\text { Aseptic loosening } \\
\text { Pain/stiffness }\end{array}$ & 4 & $\begin{array}{c}\text { Symptomatic femorotibial } \\
\text { instability in 1; severe wear of } \\
\text { the tibial polyethylene insert } \\
\text { in 3 }\end{array}$ \\
$\begin{array}{l}\text { Tibial component } \\
\text { fracture }\end{array}$ & 1 & 1 ACL rupture \\
$\begin{array}{l}\text { Infection } \\
\text { Instability }\end{array}$ & 4 & All were 2-stage revisions \\
\hline
\end{tabular}

these patients were likely more sedentary and less demanding of their knees for activity. The current knee scoring systems lose their validity when measuring patients in the third decade after surgery [15]. A TKA in which a bicruciate-retaining prosthesis is implanted is a demanding surgical procedure. If the knee is not balanced, the ACL will rupture or the tibial eminence will fracture during surgery as the knee's ROM is tested moving from flexion to extension. These procedures were performed by one surgeon (JWP) with many years of experience implanting bicruciateretaining prostheses during TKAs; thus, the results described in this report may not be reproducible in every center. Further, because of patient selection at the time of surgery, it is possible that patients with either less disease burden or with secondary stability were favored to receive the bicruciate-retaining implant. Although followup of the patients receiving implants other than the bicruciate-retaining device during the same time is not complete, almost $80 \%$ of patients did receive the implant in question. Finally, in this study, joint stability was assessed manually and may not be accurate. More sophisticated techniques to determine joint stability during and after surgery are being developed, such as sensor-guided technology [11].

In the patients in the current study, there was a high rate of implant survivorship and a low rate of complications using a bicruciate-retaining total knee prosthesis. Although implant survivorship is not the same as wellness, the functional results achieved by the patients are comparable or superior to results described in other reports using the total condylar, press-fit condylar, and meniscal-bearing prostheses $[6,15$, 20]. The ACL in the osteoarthritic knee often is degenerated macroscopically and histologically $[1,7,8]$. However, I found that with a functional ligament, degeneration was not a barrier to implanting a bicruciate-retaining prosthesis during TKA. Sixty percent or more of my patients, and as reported in other studies $[1,7,8]$, had a functionally intact ACL at presentation. The results presented here indicate a long-term 
survival rate for TKAs with this bicruciate-retaining prosthesis is similar to rates for TKAs with posterior cruciateretaining and posterior-stabilized prostheses $[3,13,18]$. In the current study, the survivorship rate is similar to the $91 \%$ survival rate in 46 knees after 12 years with the meniscalbearing bicruciate-preserving implant [6]. The Cloutier bicruciate-retaining prosthesis showed a survivorship of $82 \%$ at 20 years [21]. Kaplan-Meier survival analysis is commonly used to estimate cumulative incidence of revision after a joint arthroplasty. Because of concern in the literature regarding overestimation of revision [9], competing risk analysis is recommended in studies where death is common $[4,10]$. Since a revision cannot occur when there is death, survivorship can be overestimated with other statistical methods; therefore competing risk and Kaplan-Meier analyses were performed here.

In this study, patients achieved knee scores comparable to those reported in 20-year studies of other designs [6, 15, 20, 21]. However, some studies indicate that saving both cruciate ligaments during a TKA results in more normal kinematics compared with using posterior cruciate-retaining medial pivot and posterior-stabilized prostheses [14, $22,23]$. The mean flexion of $117^{\circ}$ at followup is consistent with flexion achieved with implants available more than 20 years ago [6, 15, 21]. Randomized comparison studies in which patients received a bicruciate-retaining knee prosthesis on one side and a posterior cruciate-retaining or substituting prosthesis on the other side have shown that $76 \%$ of patients prefer the bicruciate-retaining prosthesis $[16,18]$. Comparison studies I have done also show there is less noise, less heat generated, and less bone loss with a bicruciate-retaining prosthesis compared with other designs $[17,19]$. A TKA with a bicruciate-retaining prosthesis necessitates close approximation of the prosthesis to the anatomic contours, meticulous alignment technique, and preservation of well-balanced cruciate and collateral ligaments. A TKA in which a bicruciate-retaining prosthesis is used is a demanding surgical procedure compared with a TKA with either a posterior-cruciate or posterior-stabilized prosthesis. Preserving the ACL requires the correct ligament tension and relies on preserving the joint line, ligament balance, and alignment in all planes.

PE wear was the main mode of failure in this series (nine of 22 knees). There was a low rate of osteolysis in this series, femorotibial instability was seen twice, and late rupture of the ACL occurred once. Although the tibial component only had two stabilizing pegs and a small keel, loosening and bone loss around the prosthesis were rare in this series. The minimal constraint of the prosthesis may limit the shear on the prosthesis. Other series have shown that good long-term survival with a low incidence of osteolysis and loosening is possible using pegged tibial trays and limited constraint $[3,5]$. Patellar complications were uncommon in the current patients. It is possible that preserving the ACL is helpful for patellar stability. I did not perform a lateral patellar retinacular release in any patient. The more anatomic trochlea of the femoral component used may result in reduced forces in the patellofemoral joint [2].

I found satisfactory survivorship and function for patients receiving a bicruciate-retaining prosthesis after more than 20 years of use. Many more patients are included in the current study than in another study using bicruciate-retaining prostheses [21]. This bicruciateretaining prosthesis showed improved function, ROM, and a lower revision rate than other bicruciate-retaining prostheses that also had satisfactory long-term outcomes $[6,21]$. The bicruciate-retaining prosthesis used in this long-term study is no longer commonly used and has been replaced by more contemporary prostheses. Sacrifice of the ACL may be an unnecessary concession during TKA. However, a TKA with implantation of a bicruciateretaining prosthesis is a demanding surgical procedure. Meticulous knee balancing is necessary to avoid intraoperative ACL rupture or tibial eminence fracture when the ROM is tested moving the knee from flexion into extension. Exposure can be difficult as the tibia is not subluxed forward on the femur and less invasive techniques are not possible with a TKA with implantation of a bicruciateretaining prosthesis. Future studies using validated patient-reported outcomes should be done to determine if retention of both cruciates during TKA is appropriate in selected patients.

Acknowledgments I thank Janet L. Tremaine ELS, Tremaine Medical Communications (Dublin, OH, USA) for editorial assistance and Michael Huffman, Huffman Fisher Design (Traverse City, MI, USA) for the drawings in Fig. 1.

\section{References}

1. Allain J, Goutallier D, Voisin MC. Macroscopic and histological assessments of the cruciate ligaments in arthrosis of the knee. Acta Orthop Scand. 2001;72:266-269.

2. Andriacchi TP, Yoder D, Conley A, Rosenberg A, Sum J, Galante JO. Patellofemoral design influences function following total knee arthroplasty. J Arthroplasty. 1997;12:243-249.

3. Barrington JW, Sah A, Malchau H, Burke DW. Contemporary cruciate-retaining total knee arthroplasty with a pegged tibial baseplate: results at a minimum of ten years. J Bone Joint Surg Am. 2009;91:874-878.

4. Berry SD, Ngo I, Samelson EJ, Kiel DP. Competing risk of death: an important consideration in studies of older adults. $J \mathrm{Am}$ Geriatr Soc. 2010;58:783-787.

5. Bertin KC. Tibial component fixation in total knee arthroplasty: a comparison of pegged and stemmed designs. J Arthroplasty. 2007;22:670-678.

6. Buechel FF, Pappas MJ. Long-term survivorship analysis of cruciate-sparing versus cruciate-sacrificing knee prostheses using meniscal bearings. Clin Orthop Relat Res. 1990;260:162-169. 
7. Cushner FD, La Rosa DF, Viogorita VJ, Scuderi GR, Scott WN, Insall JN. A quantitative histologic comparison: ACL degeneration in the osteoarthritic knee. J Arthroplasty. 2003;18:687-692.

8. Douglas MJ, Hutchison JD, Sutherland AG. Anterior cruciate ligament integrity in osteoarthritis of the knee in patients undergoing total knee replacement. $J$ Orthop Traumatol. 2010;11:149-154.

9. Fennema P, Lubsen J. Survival analysis in total joint replacement: an alternative method of accounting for the presence of competing risk. J Bone Joint Surg Br. 2010;92:701-706.

10. Gooley TA, Leisenring W, Crowley J, Storer BE. Estimation of failure probabilities in the presence of competing risks: new representations of old estimators. Statist Med. 1999;18:695-706.

11. Gustke KA, Golladay GJ, Roche MW, Jerry GJ, Elson LC, Anderson CR. Increased satisfaction after total knee replacement using sensorguided technology. Bone Joint J. 2014;96:1333-1338.

12. Howell SM, Howell SJ, Kuznik KT, Cohen J, Hull ML. Does a kinematically aligned total knee athroplasty restore function without failure regardless of alignment category? Clin Orthop Relat Res. 2013;471:1000-1007.

13. Insall JN, Dorr LD, Scott RD, Scott WN. Rationale of the Knee Society clinical rating system. Clin Orthop Relat Res. 1989;248:13-14.

14. Komistek RD, Allain J, Anderson DT, Dennis DA, Goutallier D. In vivo kinematics for subjects with and without an anterior cruciate ligament. Clin Orthop Relat Res. 2002;404:315-325.
15. Patil S, McCauley JC, Pulido P, Colwell CW Jr. How do knee implants perform past the second decade? Nineteen-to 25-year followup of the press-fit condylar design TKA. Clin Orthop Relat Res. 2015;473:135-140.

16. Pritchett JW. Patient preferences in knee prostheses. J Bone Joint Surg Br. 2004;86:979-982.

17. Pritchett JW. Heat generated by knee prostheses. Clin Orthop Relat Res. 2006;442:195-198.

18. Pritchett JW. Patients prefer a bicruciate-retaining or the medial pivot total knee prosthesis. J Arthroplasty. 2011;26:224-228.

19. Pritchett JW. A comparison of the noise generated from different types of knee prostheses. J Knee Surg. 2013;26:101-104.

20. Rodriguez JA, Bhende H, Ranawat CS. Total condylar knee replacement: a 20-year followup study. Clin Orthop Relat Res. 2001;388:10-17.

21. Sabouret P, Lavoie F, Cloutier JM. Total knee replacement with retention of both cruciate ligaments: a 22 -year follow-up study. Bone Joint J. 2013:95:917-922.

22. Schmidt R, Komistek RD, Blaha JD, Penenberg BL, Maloney WJ. Fluoroscopic analyses of cruciate-retaining and medial pivot knee implants. Clin Orthop Relat Res. 2003;410:139-147.

23. Stiehl JB, Komistek RD, Cloutier JM, Dennis DA. The cruciate ligaments in total knee arthroplasty: a kinematic analysis of 2 total knee arthroplasties. J Arthroplasty. 2000;15:545-550.

24. Townley CO. Total knee arthroplasty: a personal retrospective and prospective review. Clin Orthop Relat Res. 1988;236:8-22. 